\title{
SOME CLASSES OF FUNCTIONS DEFINED BY DIFFERENCE OR DIFFERENTIAL INEQUALITIES ${ }^{1}$
}

\author{
CHARLES LOEWNER
}

1. Introduction. In the year $1934^{2} \mathrm{I}$ introduced classes of functions which I called monotonic of order $n$. The class corresponding to $n=1$ represents the functions which are monotonic in the ordinary sense. The monotonic functions of higher order are closely connected with some distinguished functions of matrices whose independent and dependent variables are real symmetric matrices of the same order.

In a recent investigation I found that the study of monotonic functions can be subordinated to the consideration of more general classes of functions which have distinguished group theoretical properties. I wish to give an account of this study.

All functions considered are supposed to be real functions of a real variable. To avoid unessential complications, I shall consider them defined in open intervals and continuous and strictly monotonically increasing there. A class $S$ of such functions will be called a transformation semigroup if it has the following properties:

(a) If $f(x) \in S$ and is defined in an interval $(a, b)$, then $f(x)$, considered only in a subinterval $\left(a^{\prime}, b^{\prime}\right) \subset(a, b)$, should also belong to $S$.

(b) If $f(x) \in S$ and $g(x) \in S$ and the composition $g[f(x)]$ can be performed, that is, if the range of $f(x)$ falls into the domain of $g(x)$, then $g[f(x)] \in S .^{3}$

(c) If $f(x)$ is a limit of functions $f_{n}(x) \in S(n=1,2,3, \cdots)$, all functions of the sequence being defined in the same interval $(a, b)$ and the convergence being uniform in any closed bounded subinterval of $(a, b)$, then $f(x) \in S$. Condition (c) represents a closure condition on $S$.

In the following the expression transformation group shall mean a class of functions which in addition to (a), (b), (c) satisfy condition $\left(\mathrm{b}^{\prime}\right)$ If $f(x) \in S$, then the inverse $f^{-1}(x) \in S$.

An address delivered before the New York meeting of the Society on April 17, 1948 , by invitation of the Committee to Select Hour Speakers for Eastern Sectional Meetings; received by the editors June 6, 1949.

1 The results of $\$ \$ 3$ and 4 were obtained while the author was under contract with the Office of Naval Research N60NR248.

${ }^{2}$ Löwner, Karl, Über monotone Matrixfunctionen, Math. Zeit. vol. 38 (1934).

${ }^{3}$ Because we do not assume that any two functions of $S$ may be composed, we might speak also of a semigroupoid. We prefer the simpler expression semigroup. 
Several semigroups have been studied in the past that have their origin in important problems of analysis. For example, the class of absolutely monotonic functions from which only the constant functions have been excluded form a transformation semigroup. A function is called absolutely monotonic if all its difference quotients and the function itself are non-negative. This class remains a transformation semigroup if the condition of non-negativity of the function itself is dropped. After this modification the semigroup contains the whole group of linear functions $a x+b(a>0)$. More extensive semigroups are obtained if the condition of non-negativity is restricted to the first $m$ difference quotients. These semigroups also contain all linear functions mentioned above.

It seems to be of great interest to study the transformation semigroups which contain completely the group of all proper projective transformations

$$
\frac{a x+b}{c x+d}, \quad\left|\begin{array}{ll}
a & b \\
c & d
\end{array}\right|>0 .
$$

(A function of (1) may be considered in any open interval not containing its pole.)

The interest in these transformation semigroups stems from the well known fact that this group cannot be extended to a Lie group of higher dimension. We shall, therefore, restrict ourselves to the study of those transformation semigroups which satisfy, besides conditions (a), (b), (c), the further condition

(d) $S$ contains the whole group of proper projective transformations.

The strictly monotonically increasing functions of a given order $n$ form a semigroup satisfying condition (d). It turns out that these semigroups have a distinguished place among the extensions of the projective group to a transformation semigroup. We shall consider them more in detail in the following section.

2. Functions monotonic of order $n$. I would like to start with a rather general generating principle of transformation semigroups: Suppose we operate in a set $\Sigma$ of elements in which a partial order is defined. That is, it can be said that of two elements $A$ and $B$ of $\Sigma$ the element $A$ is before $B$, in symbols $A \subset B$. We assume that the order relation is transitive and reflexive. That is, from $A \subset B$ and $B \subset C$ it follows that $A \subset C$ and $A \subset A$ is true for any element $A$ of $\Sigma$. Consider now a mapping

$$
X^{\prime}=F(X),
$$


where the independent and dependent variables range over some sets of $\Sigma$. A mapping (2) will be called monotonic if $A \subset B$ has $F(A)$ $C F(B)$ as consequence for any two elements $A$ and $B$ of the domain of (2) which may be compared in the sense of the given order relation.

Suppose now that there is given a homomorphism of the group of all continuous monotonically increasing functions and a group of mappings in $\Sigma$. By this the following is meant:

$(\alpha)$ To every interval $I=(a, b)$ there corresponds a set $\sigma(I)$ in $\Sigma$ such that

$$
I^{\prime} \subset I \text { implies } \sigma\left(I^{\prime}\right) \subset \sigma(I) .
$$

$(\beta)$ To a function $f(x)$ defined in $I$ there corresponds a mapping $X^{\prime}=F_{f}(X)$ with the domain $\sigma(I)$ and the range $\sigma(J)$ where $J$ is the interval which is the image of $I$ by the function $f(x)$.

$(\gamma)$ If two functions $f(x)$ and $g(x)$ can be composed then

$$
F_{o[f(x)]}=F_{o}\left[F_{f}(X)\right] .
$$

Consider now only those functions $f(x)$ which lead to monotonic mappings $F_{f}(X)$. Conditions $(\alpha),(\beta),(\gamma)$ imposed on the homomorphism insure that these functions form a class satisfying conditions (a) and (b) for transformation semigroups. By adding concepts and conditions of topological nature we could insure also that condition (c) is satisfied, but we shall omit these considerations since we shall now restrict ourselves to the special case leading to monotonic functions of higher order where (c) is almost obvious.

Take as $\Sigma$ the set of all real quadratic forms $A$ in $n$ variables $x_{1}$, $x_{2}, \cdots x_{n}$,

$$
A=\sum_{\rho, \sigma=1}^{n} a_{\rho \sigma} x_{\rho} x_{\sigma} \quad\left(a_{\rho \sigma}=a_{\sigma \rho}\right) .
$$

We may also identify $A$ with with the symmetric matrix $\left(a_{\rho \sigma}\right)$ of the coefficients. The following natural partial order may be introduced in $\Sigma$ : We say that $A \subset B$ if

$$
\sum_{\rho, \sigma=1}^{n} a_{\rho \sigma} x_{\rho} x_{\sigma} \leqq \sum_{\rho, \sigma=1}^{n} b_{\rho \sigma} x_{\rho} x_{\sigma}
$$

for all values of the variables $x_{i}$. One may also say that the form $B-A$ is non-negative definite. Consider now a function $f(x)$ defined in an open interval $(a, b)$ and a quadratic form $X$ whose characteristic values $\lambda_{i}$ all fall into $(a, b)$. As usual we mean by $f(X)$ that quadratic form which has the same characteristic vectors as $X$ but the new characteristic values $f\left(\lambda_{i}\right)$. If, for example, $f(x)$ is a polynomial $f(x)$ 
$=\sum_{\rho=0}^{m} a_{\rho} x^{\rho}$, then $f(X)=\sum_{\rho=0}^{m} a_{\rho} X^{\rho}$. The domain of the matrix function $f(X)$ consists of all the symmetric matrices whose characteristic values fall into $(a, b)$. If we now set

$$
F_{f}(X)=f(X) \text {, }
$$

a correspondence $f \rightarrow F$ is obtained satisfying all the conditions defining a homomorphism. We obtain a definite semigroup $S_{n}$ if we select those functions $f(x)$ which lead to a monotonic matrix function in the space of symmetric matrices of degree $n$. The functions of $S_{n}$ are called montonic of order $n$.

Monotonicity of order 1 means evidently monotonicity in the ordinary sense. Further it is easily seen by considering reduced matrices

$$
A=\left(\begin{array}{ll}
B_{m} & 0 \\
0 & C_{n-m}
\end{array}\right)
$$

where $B_{m}$ and $C_{n-m}$ are symmetric matrices of degrees $m$ and $n-m$ respectively, that

$$
S_{n} \subset S_{m}
$$$$
(m<n) \text {. }
$$

In other words, the monotonicity of order $n$ implies monotonicity of any lower order.

The fundamental properties of monotonic functions of higher order will now be formulated in several theorems. ${ }^{4}$

THEOREM 1. In order that a function $f(x)$ be monotonic of order $n$ in the interval $(a, b)$ it is necessary and sufficient that for any $2 m$ values

$$
a<\xi_{1}<\eta_{1}<\xi_{2}<\eta_{2}<\cdots<\xi_{m}<\eta_{m}<b \quad(m \leqq n)
$$

the inequality

$$
\operatorname{det}\left|K\left(\xi_{i}, \eta_{k}\right)\right|_{i, k=1}^{m} \geqq 0
$$

holds, where $K(\xi, \eta)=(f(\xi)-f(\eta)) /(\xi-\eta)$ represents the difference quotient of $f(x)$.

For $n=2$ these conditions have a simple meaning. They say that if $f(x)$ is not constant it is strictly monotonically increasing and that for any four values $x_{1}<x_{2}<x_{3}<x_{4}$ from $(a, b)$ and the corresponding values $f\left(x_{1}\right)<f\left(x_{2}\right)<f\left(x_{3}\right)<f\left(x_{4}\right)$ the inequality

$$
\left(f\left(x_{1}\right), f\left(x_{2}\right), f\left(x_{3}\right), f\left(x_{4}\right)\right) \geqq\left(x_{1}, x_{2}, x_{3}, x_{4}\right)
$$

${ }^{4}$ See paper [1] and the doctoral thesis of O. Dobsch, Matrixfunctionen beschränkter Schwankung, Math. Zeit., vol. 43 (1937). 
holds. The expression $\left(u_{1}, u_{2}, u_{3}, u_{4}\right)=\left(u_{1}-u_{3}\right)\left(u_{2}-u_{4}\right) /\left(u_{1}-u_{4}\right)\left(u_{2}-u_{3}\right)$ designates as usual the cross-ratio of the four values $u_{1}, u_{2}, u_{3}, u_{4}$.

A simple consequence of this inequality is the fact that a function which, along with its inverse, is monotonic of order 2 must be a projective one.

From the fundamental Theorem 1, differential properties of the monotonic functions can be derived. They are expressed in the following four theorems.

THEOREM 2. A monotonic function $f(x)$ of order $n>1$ is of class $C_{2 n-3}$ (that is, $(2 n-3)$ times continuously differentiable) and its $(2 n-3) r d$ derivative is a convex function. The derivatives $f^{(2 n-2)}(x)$ and $f^{(2 n-1)}(x)$ exist, therefore, almost everywhere. For nonconstant $f(x)$ the first derivative $f^{\prime}(x)>0$.

In the following, by convergence of a sequence of functions it is understood that the functions are defined in the same interval $(a, b)$ and that the convergence is uniform in any closed bounded part of $(a, b) .{ }^{5}$ With this in mind we formulate the following theorems.

Theorem 3. A monotonic function of order $n>1$ is the limit of a sequence of monotonic functions of order $n$ all belonging to class $C_{2 n-1}$.

Theorem 4. A function $f(x)$ of class $C_{2 n-1}$ in $(a, b)$ is monotonic of order $n$ there if and only if the quadratic form

$$
\sum_{i, k=1}^{n} \frac{f^{(i+k-1)}(x)}{(i+k-1) !} \xi_{i} \xi_{k} \geqq 0
$$

for all $x$ in $(a, b)$.

THEOREM 5. If $a_{i}(i=1, \cdots, 2 n-1)$ are values such that the quadratic form

$$
\sum_{i, k=1}^{n} a_{i+k-1} \xi_{i} \xi_{k}>0
$$

then there exists a monotonic function $f(x)$ of order $n$ and of class $C_{2 n-1}$ such that at some preassigned point $x_{0}$

$$
\frac{1}{i !} f^{(i)}\left(x_{0}\right)=a_{i} \quad(i=1, \cdots, 2 n-1) .
$$

It is of great interest to give a characterization of the functions which are monotonic of arbitrarily high order. They also form a semigroup, say $S_{\infty}$, which is contained in every $S_{n}$. A complete answer to this problem can be given by combining the preceding theorems with

\footnotetext{
s This convergence is sometimes called "normal" convergence.
} 
some results of the theory of moments. From Theorem 2 we conclude that a function belonging to $S_{\infty}$ is differentiable of arbitrarily high order and (11) holds, therefore, for any $n$. From this we can derive by using results from the theory of moments the following theorem.

THEOREM 6. $A$ (nonconstant) function $f(x)$ defined in $(a, b)$ belongs to $S_{\infty}$ if and only if it is analytic in $(a, b)$, can analytically be continued into the whole upper half plane, and represents there an analytic function $f(z)$ of $z=x+i y$ whose imaginary part is positive. (Examples are given by the functions $x^{\mu}(0<\mu \leqq 1)$ and $\log x$ for $x>0$.)

It is natural to ask whether a still more restricted class of functions forming a semigroup may be obtained by considering, instead of quadratic forms in finite dimensions, bounded self-adjoint operators in Hilbert space. The order definition can evidently be taken over from the definition in finite dimensions. It is also well known how to define $f(X)$ if the spectrum of $X$ falls into the domain where $f(x)$ is defined. But it turns out that the functions $f(x)$ leading to monotonic functions in the operator space form the full semigroup $S_{\infty}$.

3. Transformation semigroups containing the proper projective group which may be defined by differential inequalities. The contents of Theorems 3, 4, and 5 suggest a new description of the semigroup of nonconstant monotonic functions of order $n$ which brings it into closer connection with a Lie group.

If two functions $f(x)$ and $g(x)$ both belonging to $C_{m}$ are combined, $f[g(x)]=h(x)$, then the derivatives of $h(x)$ up to order $m$ can be computed from the derivatives of the composing functions $f(x)$ and $g(x)$, up to the same order. If, for $u=g(x)$,

$$
\frac{f^{(i)}(u)}{i !}=a_{i}, \quad \frac{g^{(i)}(x)}{i !}=b_{i}, \quad \text { and } \quad \frac{h^{(i)}(x)}{i !}=c_{i} \quad(i=1,2, \cdots, m)
$$

then

$$
\begin{aligned}
c_{1}= & a_{1} b_{1} \\
c_{2}= & a_{1} b_{2}+a_{2} b_{1}^{2} \\
\ldots \ldots \ldots \ldots & \ldots \ldots \ldots \ldots \\
c_{m}= & a_{1} b_{m}+a_{2}\left[\sum_{\alpha_{1}+\alpha_{2}=m}\left(b_{\alpha_{1}} b_{\alpha_{2}}\right)\right]+a_{3}\left[\sum_{\alpha_{1}+\alpha_{2}+\alpha_{2}=m}\left(b_{\alpha_{1}} b_{\alpha_{2}} b_{\alpha_{2}}\right)\right] \\
& +\cdots+a_{m} b_{1}^{m} .
\end{aligned}
$$

Equations (13) can be interpreted as a composition rule for ordered 
pairs of points $\left(a_{1}, a_{2}, \cdots, a_{m}\right)$ and $\left(b_{1}, b_{2}, \cdots, b_{m}\right)$ of the $m$-dimensional Euclidean space $E_{m}$. As is well known, this composition rule defines a Lie group $l_{m}$ if only points whose first coordinate is positive are admitted.

By Theorems 4 and 5 a subset of $l_{2 n-1}$ is distinguished; namely, the set of points $\left(a_{1}, a_{2}, \cdots, a_{2 n-1}\right)$ of $l_{2 n-1}$ for which (12) is satisfied. Call its closure $s_{2 n-1}$. From the named theorems we immediately derive the fact that the composition rule (13) applied to any pair of points of $s_{2 n-1}$ leads again to a point of $s_{2 n-1}$. To facilitate the description of $s_{2 n-1}$ we introduce the definition: A subset of a topological group will be called a subsemigroup of it if it represents a closed set and if it is closed under the composition rule of the group.

We now can say that $s_{2 n-1}$ represents a subsemigroup of $l_{2 n-1}$.

The transformation semigroup of monotonic functions of order $n$ can be derived from $s_{2 n-1}$ by the following procedure: Consider all functions $f(x)$ of class $C_{2 n-1}$ such that the point

$$
\left(\frac{f^{\prime}(x)}{1 !}, \frac{f^{(2)}(x)}{2 !}, \ldots, \frac{f^{(2 n-1)}(x)}{(2 n-1) !}\right)
$$

falls into $s_{2 n-1}$ for all $x$ where $f(x)$ is defined. The transformation semigroup $S_{n}$ consists now of all functions thus defined and their limits.

This characterization of $S_{n}$ leads to a more general principle of construction of transformation semigroups. Take an arbitrary subsemigroup $s$ of $l_{m}$. In the same way as from $s_{2 n-1}$ a class of functions can be derived from $s$ by considering those $f(x)$ of class $C_{m}$ such that

$$
\left(\frac{f^{(1)}(x)}{1 !}, \frac{f^{(2)}(x)}{2 !}, \ldots, \frac{f^{(m)}(x)}{m !}\right)
$$

always belongs to $s$, and adding to these functions their limits. It is easily seen that they form a transformation semigroup. We thus arrive at a method of constructing a transformation semigroup from an arbitrary subsemigroup of $l_{m}$. If, furthermore, all elements (14) derived from a transformation of this semigroup of class $C_{m}$ belong to $s$, we shall say that the semigroup is characterized by or belongs to $s$.

We are particularly interested in transformation semigroups which contain the proper projective group. In order that the transformation semigroup characterized by a subsemigroup $s$ of $l_{m}$ have this property, it is easily seen that $s$ must contain the elements

$$
a_{i}=p \alpha^{i-1} \quad(i=1, \cdots, m) \quad(p>0, \alpha \text { arbitrary }) .
$$

We shall call the elements (14) the projective elements of $l_{m}$. We require 
therefore that $s$ contain all projective elements of $l_{m}$.

The investigation of subgroups of a Lie group is essentially facilitated by the introduction of the infinitesimal viewpoint. It is a well known fact that a closed connected subgroup of a Lie group $g$ is completely determined by its infinitesimal elements. It is, therefore, appropriate to ask whether a subsemigroup $s$ of $g$ can be connected in a similar way with a system of infinitesimal elements and also how far the subsemigroup is determined by them.

The definition of an infinitesimal element of $s$ if the unit element $e$ of $g$ is an accumulation point of $s$ can be introduced exactly in the same way as in the case of a subgroup: Take an arbitrary sequence of elements $a_{n}$ of $s$ different from the unit element $e$ of $g$ but converging to $e$. Any vector $\alpha$ at $e$ whose direction is a limiting direction of the segments $e a_{n}$ will be called an infinitesimal element of $s$.

According to this definition the following lemma holds.

LEMma 1. Any multiple c $\alpha(c \geqq 0)$ of an infinitesimal element $\alpha$ of $s$ is also an infinitesimal element of $s$.

The next two lemmas on infinitesimal elements of a subsemigroup can be proved in the same manner as in the case of a subgroup.

LEMмA 2. The sum of two infinitesimal elements of $s$ is also an infinitesimal element of $s$.

Lемма 3. The infinitesimal elements of $s$ form a closed set.

For a subgroup multiplication of infinitesimal elements with arbitrary real factors is allowed. The infinitesimal elements of a subgroup form therefore a vector space. (Its dimension coincides with the dimension of the group.) This is not the case for a semigroup. But, as a result of our lemmas the following theorem holds.

THEOREM 7. The infinitesimal elements of $s$ form a closed convex set of vectors invariant under multiplication with an arbitrary positive factor.

We shall call the totality of the infinitesimal elements of $s$ the infinitesimal cone of $s$ and designate it with $\sigma_{s}$.

A fundamental means of investigation of Lie groups is the generation of elements from infinitesimal elements. As was stated before, each connected subgroup of a Lie group can be completely generated from its infinitesimal elements. By the same methods used in Lie group theory the following theorem regarding subsemigroups can be proved.

THEOREM 8. Each element of a Lie groupl generated from infinitesimal 
elements of a subsemigroup s of $l$ also belongs to $s$. The elements obtained in this way together with their limiting elements form again a subsemigroup $\bar{s}$ of $l$.

In general $\bar{s}$ will not coincide with $s$ as will soon be shown by examples.

The following further slight generalization of a well known theorem of Lie groups will shortly be used.

THEOREM 9. Let $l$ be a Lie group, $s$ a subsemigroup of $l$, and $a$ an element of $l$ which with its inverse belongs to $s$. Then the transform $a \alpha a^{-1}$ of any infinitesimal element $\alpha$ of $s$ also represents an infinitesimal element of $s$.

After stating these general theorems we return to the special group $l_{m}$. We are interested in finding all subsemigroups $s$ of $l_{m}$ satisfying condition (a): s shall contain all projective elements of $l_{m}$.

The cases $m=1$ and $m=2$ are of no interest since there evidently do not exist proper subsemigroups of this kind. We assume therefore from now on that $m \geqq 3$.

A slight restriction will further be added resulting from the fact that we are interested only in semigroups leading to transformation semigroups which are proper extensions of the proper projective group. This has as consequence that there must exist an element $a=\left(a_{1}, a_{2}, \cdots, a_{m}\right)$ of $s$ such that $\left(a_{1}, a_{2}, a_{3}\right)$ is not a projective element of $l_{3}$. Otherwise each function $f(x)$ of class $C_{3}$ belonging to the corresponding transformation semigroup would satisfy the differential equation $f^{\prime} f^{\prime \prime \prime} / 3 !=\left(f^{\prime \prime} / 2 !\right)^{2}$ whose solutions are the projective transformations. In order to obtain proper extensions of the projective group we have therefore to introduce the condition (b): $s$ shall contain elements such that

$$
a_{1} a_{3}-a_{2}^{2} \neq 0 \text {. }
$$

A first remark about the structure of $s$ is contained in the following easily proved theorem.

Theorem 10. A subsemigroup $s$ of $l_{m}$ satisfying conditions (a) and (b) represents a connected subset of $l_{m}$.

Let us now consider the infinitesimal cone $\sigma_{s}$ of $s$.

An infinitesimal element of $l_{m}$ is represented by a vector $\alpha$ $=\left(\alpha_{1}, \alpha_{2}, \cdots, \alpha_{m}\right)$ in $m$ dimensions. In particular the infinitesimal elements of the group of the projective elements of $l_{m}$ have the form

$$
\pi=\left(\alpha_{1}, \alpha_{2}, 0,0, \cdots, 0\right) \quad\left(\alpha_{1}, \alpha_{2} \text { arbitrary }\right)
$$


The following important theorem can now be derived.

THEOREM 11. At least one of the elements

$$
\omega=(0,0,1,0, \cdots), \quad-\omega=(0,0,-1,0, \cdots)
$$

belongs to the infinitesimal cone $\sigma_{8}$ of $s$.

The proof is obtained by use of suitable transforms of an element satisfying condition (16) with projective elements.

Assume now, for example, that $\sigma_{s}$ contains $\omega$. New elements of $\sigma_{s}$ can now be obtained by use of Theorem 9 , which implies also that any transform $p^{-1} \omega p$ of $\omega$ with an arbitrary projective element $p$ must belong to $\sigma_{s}$. With the aid of Lemmas 1 and 2 we may say that all sums of the form

$$
\sum_{\rho=1}^{N} p_{\rho}^{-1} \omega p_{\rho}
$$

where the $p_{\rho}(\rho=1,2, \cdots, N)$ represent arbitrary projective elements, are elements of $\sigma_{s}$.

If the computation is performed one finds that the first two components of (19) are zero and the following $(m-2)$ components are the first $(m-2)$ moments of $N$ non-negative masses. According to an important result of the moment problem these systems of numbers and their limits can be characterized by the non-negativity of the quadratic form

$$
\sum_{\rho, \sigma=1}^{[(m-1) / 2]} \alpha_{\rho+\sigma+1} \xi_{\rho} \xi_{\sigma} .
$$

To the elements thus obtained we can add an arbitrary projective element (17) without leaving $\sigma_{s}$. Call the totality of the elements obtained $\tau_{m}$.

If we had started with $-\omega$ instead of $\omega$, we would have obtained the result that the set $-\tau_{m}$ of elements making (20) nonpositive belongs completely to $\sigma_{s}$. We arrive therefore at the following theorem.

THEOREM 12. The infinitesimal cone $\sigma_{s}$ of a subgroup $s$ of $l_{m}$ satisfying conditions (a) and (b) must completely contain either $\tau_{m}$ or $-\tau_{m}$.

We now make the important observation that $\tau_{m}$ and $-\tau_{m}$ are themselves the infinitesimal cones of subsemigroups of $l_{m}$ satisfying conditions (a) and (b). To show this we go back to the inequalities (12) characterizing the monotonic functions of order $n$. It easily leads to:

THEOREM 13. The infinitesimal cone of the semigroup defining the 
monotonic functions of order $n$ consists of the infinitesimal elements of $l_{2 n-1}$ that make form (20) non-negative.

Theorem (13) proves our assertion for odd values of $m$. For even dimension the proof is furnished by the following theorem.

TheOREM 14. If $m$ is an even number, the elements $\left(a_{1}, a_{2}, \cdots, a_{m}\right)$ which can be extended to elements $\left(a_{1}, a_{2}, \cdots, a_{m+1}\right)$ belonging to $s_{m+1}$ form a semigroup themselves and the corresponding infinitesimal cone is $\tau_{m}$.

Theorems 12, 13, and 14 lead now to the fundamental theorem.

Theorem 15. The infinitesimal cones $\tau_{m}$ and $-\tau_{m}$ play a distinguished role among the infinitesimal cones of subsemigroups of $l_{m}$ satisfying conditions (a) and (b). This role is expressed by the minimum property: Each of these cones contains either $\tau_{m}$ or $-\tau_{m}$.

Theorem 15 can be sharpened by:

THEOREM 15'. If an infinitesimal cone $\sigma$ satisfying conditions (a) and (b) contains both cones $\tau_{m}$ and $-\tau_{m}$, then it consists of all infinitesimal elements of $l_{m}$.

The proof follows almost immediately from the fact that $\tau_{m}$ contains $m$ linearly independent elements. If both $\tau_{m}$ and $-\tau_{m}$ belong to $\sigma$, any linear combination with arbitrary real coefficients of these elements must belong to $\sigma$.

It is possible to describe a procedure by which all infinitesimal cones of subsemigroups of $l_{m}$ satisfying conditions (a) and (b) can be constructed: Take an arbitrary infinitesimal element $\alpha$ such that $\alpha_{3} \neq 0$ and form all transforms

$$
p \alpha p^{-1}
$$

with projective elements $p$ of $l_{m}$. Next, form the least closed convex set containing all elements (21) and all infinitesimal projective elements. Call it $C(\alpha)$. Then the following theorem holds.

THEOREM 16. The sets $C(\alpha)$ are infinitesimal cones of subsemigroups of $l_{m}$ satisfying conditions (a) and (b). One obtains the most general such cone belonging to a proper subsemigroup of $l_{m}$ by forming the least convex closure of any union of cones $C(\alpha)$ belonging to elements $\alpha$ whose third components $\alpha_{3}$ have the same sign.

After the possible infinitesimal cones have been described, the problem remains to determine the subsemigroups to which they belong. 
As was already mentioned before, the problem of constructing semigroups from their infinitesimal cones presents greater difficulties than the corresponding problem for subgroups of Lie groups. This is due to the fact that two different subsemigroups of a Lie group, even if they are represented by connected sets, may have the same infinitesimal cone. This is already true of our $\tau_{m}$, to which our primary interest is directed, for $m \geqq 5$.

According to Theorem 8, there exists for each infinitesimal cone, $\sigma_{s}$, a least subsemigroup $\bar{s}\left(\sigma_{s}\right)$. It consists of the elements of the Lie group under consideration that can be generated by the elements of $\sigma_{s}$, and their limiting elements. If we now compare the subsemigroups $s_{m}$ of $l_{m}$ derived from the monotonic functions of higher order with the least semigroup $\bar{s}\left(\tau_{m}\right)$ having the same infinitesimal cone $\tau_{m}$, then the result is obtained: For $m=1,2,3,4, \bar{s}\left(\tau_{m}\right)=s_{m}$. For $m \geqq 5, \bar{s}\left(\tau_{m}\right)$ is a proper subsemigroup of $s_{m}$.

It can be shown that the transformation semigroup $T_{m}$ generated by $\bar{s}\left(\tau_{m}\right)$ is characterized by $\bar{s}\left(\tau_{m}\right)$. This leads to the following theorem.

THEOREM 17. Among the transformation semigroups which are proper extensions of the projective group and which are characterized by subsemigroups of $l_{m}$, there exist two distinguished ones, $T_{m}$ and $T_{m}^{\prime}, T_{m}^{\prime}$ consisting of the inverse transformations of $T_{m}$, having the minimum property: Each such semigroup contains either $T_{m}$ or $T_{m}^{\prime}$ completely.

4. Construction of minimal transformation semigroups including the proper projective group as proper subgroups. The discovery of the minimum property of the semigroups $T_{m}$ leads us to the following conjecture:

Among the proper extensions of the proper projective group to a transformation semigroup there exist two distinguished ones (call them $T$ and $T^{\prime}, T^{\prime}$ being formed by the inverses of the transformations of $T$ ) characterized by the following property: Each such transformation semigroup contains either $T$ or $T^{\prime}$ completely. If it contains nonprojective transformations from both $T$ and $T^{\prime}$, then it is the group of all continuous transformations.

I could verify this conjecture under the restrictive assumption that only transformation semigroups are considered that contain also nonprojective transformations of class $C_{3}$.

$T$ can be characterized in the following way: It consists of those monotonic transformations of arbitrarily high order which can be analytically continued into the total upper half plane and yield schlicht mappings of the latter into itself. ${ }^{6}$

Syracuse UnIVERSITY

${ }^{6}$ The proofs of the theorems of $\$ \S 3$ and 4 will be given in another paper. 